\title{
Calidad educativa y satisfacción del estudiante en la Facultad de Ciencias Económicas y Administrativas de la Universidad Adventista de Bolivia
}

\author{
Educational quality and student satisfaction in the Faculty of Economic and Administrative \\ Sciences of the Adventist University of Bolivia \\ Rubén Choque Mamani ruben.choque@uab.edu.bo \\ Código ORCID: 0000-0002-6956-0394 \\ Universidad Adventista de Bolivia, Cochabamba, Bolivia
}

Recibido octubre 2020 | Arbitrado noviembre 2020 | Aceptado en diciembre 2020| Publicado enero 2021

\section{Resumen}

Palabras clave:

Calidad educativa;

satisfacción del estudiante; criterios

de evaluación
La calidad educativa en la educación superior es una temática de gran interés para las universidades y para el Estado de Bolivia. El objetivo de la investigación fue determinar el nivel alcanzado en los factores y características que contribuyen con la calidad académica, de la Facultad de Ciencias Económicas y Administrativas de la Universidad Adventista de Bolivia, desde la satisfacción de los estudiantes del plan de estudios 2016-2020. Se desarrolló el estudio con un enfoque cuantitativo y diseño no experimental explicativo. Se seleccionó una muestra aleatoria de 255 estudiantes. La técnica para la recolección de los datos fue la encuesta y el instrumento que se construyó para este estudio fue un cuestionario estructurado de respuesta tipo escala de Likert, con cinco posibles respuestas. El hallazgo más relevante es que los factores y características que contribuyen con la calidad educativa se ubican en el Nivel 3, la calidad educativa es razonable con respecto al modelo considerado como deseable.

\section{Abstract}

Keywords:

Educational quality; student satisfaction; evaluation criteria
The educational quality in higher education is a subject of great interest for the universities and for the State of Bolivia The objective of the research was to determine the level reached in the factors and characteristics that contribute to the academic quality, of the Faculty of Economic Sciences and Administrative of the Adventist University of Bolivia, from the satisfaction of the students of the 2016-2020 curriculum. The study was developed with a quantitative approach and an explanatory non-experimental design. A random sample of 255 students was selected. The technique for data collection was the survey and the instrument that was constructed for this study was a structured questionnaire with a Likert scale response, with five possible responses. The most relevant finding is that the factors and characteristics that contribute to educational quality are located at Level 3, educational quality is reasonable with respect to the model considered desirable. 


\section{INTRODUCCIÓN}

La calidad educativa universitaria es un constructo que genera constantemente investigación desde diferentes campos como lo son el educativo, gerencial, socio económico y político. Cada uno de estos campos tiene interés por la calidad educativa por diferentes razones. La Universidad como institución educativa tiene como misión que sus egresados reúnan los perfiles deseables de egreso que les permitan desempeñarse de manera óptima en las actividades típicas de su profesión y además impactar de forma positiva en la sociedad; para ello necesita una educación de calidad, pero esto es más fácil declararlo que hacerlo tangible en la realidad. Una de las razones es que el término de calidad de la enseñanza superior es un concepto pluridimensional y por lo tanto complejo, que exige conocer todas sus funciones y actividades como la enseñanza, programas académicos, investigación, personal, estudiantes, infraestructura, edificaciones, instalaciones equipamiento y servicios a la comunidad $y$ al mundo universitario (Unesco, 2009).

Para describir, comparar, analizar o evaluar la calidad educativa en la universidad se debe decidir quiénes son los que pueden determinar el grado o nivel de esta variable, al respecto Lago, López, Municio, Ospina y Vergara (2013) resaltan la importancia de la satisfacción del estudiante, por ello la calidad sería el resultado de comparar las expectativas que tiene el usuario con la percepción del servicio recibido; en otras palabras la calidad está relacionada con la satisfacción que muestran los estudiantes del servicio y el valor de lo que reciben.
La calidad debe ser definida en términos que faciliten la percepción y captación en los procesos de evaluación y acreditación universitaria, debe incluir las funciones sustantivas de la universidad: la docencia, la investigación la proyección social y la gestión administrativa.

Lago de Vergara, Gamoba y Montes (2014) destacan que la Declaración o Tratado de Bolonia en 1999, es una referencia importante para la investigación en calidad educativa universitaria, ya que este tratado impactó la educación superior en Europa con importantes efectos en América Latina y el Caribe. El impacto del tratado de Bolonia se observa actualmente en Latinoamérica en la participación del estudiante en su formación, una nueva forma de comprender las formas de aprender y la cuantificación del aprendizaje a través de los créditos.

En este mismo orden de ideas Brunner (2008) resalta que la influencia del tratado de Bolonia, en la educación universitaria en América Latina se puede evidenciar en la importancia que se le asigna a la formación de los docentes, la movilidad de los docentes en su campo laboral y la movilidad de los estudiantes, así como también entender el aprendizaje por competencias.

En el contexto nacional, las instituciones educativas del nivel superior en Bolivia, han ingresado a un proceso de autoevaluación y acreditación por parte del Ministerio de Educación. En la Facultad de Ciencias Económicas de la Universidad Adventista se considera la calidad educativa como un compromiso con sus estudiantes y con la sociedad; siempre en pro de una educación de calidad, inclusiva, cristiana, fraterna y equitativa para lograr la formación de un profesional que logre imprimir estos valores en el ambiente en el cual se desempeñe. 
Con respecto a los valores cabe citar a Luna (1997) quien le da importancia al ethos organizativo, el cual está constituido por el entramado coherente de valores individuales y grupales compartidos en una organización. Estos valores orientan las decisiones, refuerzan los compromisos, y le da sentido a todas las acciones de los actores involucrados en la universidad.

En la actualidad, es una necesidad contar con educación de calidad, al respecto Alarcón (2008) sustenta

La exigencia de contar con una educación de mayor calidad es una demanda de la sociedad actual, un imperativo del exigente mundo en que estamos inmersos, el cual ha creado la urgente necesidad de que el trabajo del hombre sea mucho más eficiente, para lo cual requiere de mayor preparación. (p. 32)

Para asegurar la calidad de la educación superior se deben realizar procesos de evaluación para identificar las debilidades y las fortalezas con el fin de ajustar constantemente los distintos procesos educativos, gerenciales, tecnológicos.

Las experiencias relacionadas con la evaluación de la calidad son alentadoras, hay buenas referencias tanto en Europa como en América. En Sur América, el Sistema de Acreditación Regional de Carreras Universitarias es el resultado de un Acuerdo entre los Ministros de Educación de Argentina, Brasil, Paraguay, Uruguay, Bolivia y Chile, homologado por el Consejo del Mercado Común del MERCOSUR (2008) a través de la Decisión CMC ํํ17/08.
El Sistema de Acreditación Regional de Carreras Universitarias para el MERCOSUR (ARCU-SUR) es un sistema de acreditación de la calidad de la formación de nivel universitario que está orientado a obtener una mejora permanente en la formación de las personas, siguiendo patrones de calidad requeridos para la promoción del desarrollo económico, social, político y cultural de la zona del MERCOSUR.

Los gobiernos exigen a las instituciones acciones dirigidas a mejorar la calidad esto ha incrementado la implementación de proyectos de evaluación institucional. Por esta razón en Centro y Sur América, desde 1989 hasta nuestros días, han surgido organismos para regular la acreditación de la educación superior y la evaluación de la calidad educativa, entre ellos: la Comisión Nacional para la Evaluación de la Educación Superior (CONAEVA) en México, el Consejo Superior en Chile, el Consejo Nacional de Acreditación (CNA) en Colombia, la Comisión Nacional de Evaluación y Acreditación Universitaria (CONEAU) en Argentina, el Mecanismo Experimental de Acreditación de Carreras para el Reconocimiento de Grado Universitario para MERCOSUR, la Comisión Nacional Intersectorial de Aseguramiento de la Calidad de la Educación Superior (CONACES) en Colombia.

Fernández (2005) reseña que la experiencia desarrollada por los países en materia de evaluación y acreditación, tanto institucional como de programas, ha implicado para las instituciones la asimilación de una cultura de la evaluación y de la calidad, lo que ha permitido que se miren a su interior, se confronten frente a 
parámetros previamente establecidos $\mathrm{y}$ definan mecanismos de mejoramiento. Asimismo, en la mayoría de los países los resultados de los procesos se orientan, bien de manera interna para el aprendizaje institucional y el mejoramiento de la calidad, o bien de manera externa, para dar información y garantía a la sociedad sobre el servicio que se ofrece o para orientar acciones del Estado para fines de financiamiento.

Para la Facultad de Ciencias Económicas y Administrativas (FCEA) de la Universidad Adventista de Bolivia (UAB), es necesaria la evaluación para que la formación que pretende se produzca con la mayor calidad, por lo tanto, identificar cuáles son los factores que contribuyen en el logro de la calidad educativa en el plan de estudios 2016 - 2020, permitirá encontrar fortalezas y debilidades, ayudando estas en la planificación de las próximas gestiones.

El problema de estudio queda reflejado en la interrogante de investigación: ¿Cuál es el nivel alcanzado en los factores $y$ características que contribuyen con la calidad educativa, de la FCEA de la UAB, desde la satisfacción de los estudiantes del plan de estudios 2016-2020?

De la misma manera, el objetivo que guía esta investigación es determinar el nivel alcanzado en los factores $y$ características que contribuyen con la calidad académica, de la FCEA de la UAB, desde la satisfacción de los estudiantes del plan de estudios 2016-2020.

Son ocho los factores para el proceso de evaluación: Misión y Proyecto Institucional, Estudiantes, Profesores, Procesos académicos, Bienestar institucional, Organización, administración y gestión, Egresados e impacto sobre el medio,
Recursos físicos y financieros. Dentro de cada factor están determinadas las características centrales en el servicio educativo, que suman a 43 características que serán evaluadas por los estudiantes.

La hipótesis planteada en este estudio es: El nivel promedio alcanzado en los factores y características que contribuyen con la calidad educativa de la FCEA de la $\mathrm{UAB}$, desde la satisfacción de los estudiantes del plan de estudios 2016 - 2020, está en el Nivel 3 entre 56 - 70, se cumple aceptablemente (Calidad como razonable con respecto al modelo considerado deseable). La hipótesis será aceptada si se encuentra en la escala del Nivel 3 o superior y rechazada en caso de ser inferior.

\section{MÉTODO}

El enfoque es cuantitativo, de acuerdo al objetivo es una investigación explicativa ya que busca determinar los factores $y$ características de la calidad educativa, el diseño es de campo no experimental porque los datos fueron recolectados en el contexto natural, la Universidad Adventista de Bolivia.

La población de estudio fue de 342 estudiantes de la Facultad de Ciencias Económicas y Administrativas, matriculados desde el tercer semestre, egresados y titulados al 2020. La muestra fue aleatoria de 255 estudiantes que estuvieron disponibles para responder el instrumento; 121 estudiantes de Contaduría, 118 de Administración y 16 de Comercial.

La técnica para la recolección de los datos fue la encuesta y el instrumento que se construyó para este estudio fue un cuestionario estructurado de respuesta tipo escala de Likert, con cinco posibles respuestas: Se cumple plenamente 
(5), Se cumple en alto grado (4), Se cumple aceptablemente (3), Se cumple insatisfactoriamente (2) y No se cumple (1).

Para interpretar los resultados se siguieron los siguientes parámetros:

Nivel 5 (Se cumple plenamente), si el porcentaje oscila entre 86 - 100, se considera la calidad como satisfacción total con respecto al modelo considerado deseable.

Nivel 4 (Se cumple en alto grado), si el porcentaje oscila entre $71-85$, se considera la calidad con menor distancia con respecto al modelo considerado deseable.

Nivel 3 (Se cumple aceptablemente), si el porcentaje oscila entre 56 - 70, se considera la calidad como razonable con respecto al modelo considerado deseable.

$$
\begin{array}{llll}
\text { Nivel } & 2 & \text { (Se } & \text { cumple }
\end{array}
$$

insatisfactoriamente), si el porcentaje oscila entre $31-55$, se considera la calidad con mayor distancia con respecto al modelo considerado deseable.

Nivel 1 (No se cumple), si el porcentaje oscila entre 1 - 30, se considera la calidad como insatisfacción total con respecto al modelo considerado deseable.

Los 8 factores y 43 características que constituyen el instrumento se resumen en el Cuadro 1.

Cuadro 1. Modelo de evaluación

Factor Características

1. Misión y Proyecto institucional

2. Estudiantes

3. Profesores

4. Procesos académicos

5. Bienestar institucional

6. Organización, administración y gestión

7. Egresados e impacto sobre el medio

8. Recursos físicos y financieros
Misión institucional, proyecto institucional, proyecto educativo del programa, pertinencia del programa

Mecanismos de ingreso, número de admitidos, permanencia y deserción, participación en formación integral, competencias, reglamento, evaluación

Selección, estatuto, número, dedicación, nivel de formación, desarrollo, interacción, estímulos, producción de material, remuneración

Integralidad del currículo, flexibilidad del currículo, interdisciplinariedad, relaciones del programa, metodologías de enseñanza, trabajos de los estudiantes, evaluación y autorregulación del programa, investigación formativa, compromiso con la investigación, extensión o proyección social, recursos bibliográficos, recursos informáticos, recursos de apoyo didáctico

Políticas, programas y servicios de bienestar universitario,

organización, administración y gestión del programa, sistemas de comunicación e información, dirección del programa, promoción del programa,

Influencia del programa en el medio, seguimiento de los egresados, impacto de los egresados en el medio social y académico

Recursos físicos, presupuesto del programa, administración de recursos 
RESULTADOS

En primer lugar se presentan las características demográficas de la muestra, las siglas utilizadas para las especialidades son: Contabilidad (LCP), Administración (LANI) y Comercial (LIC).

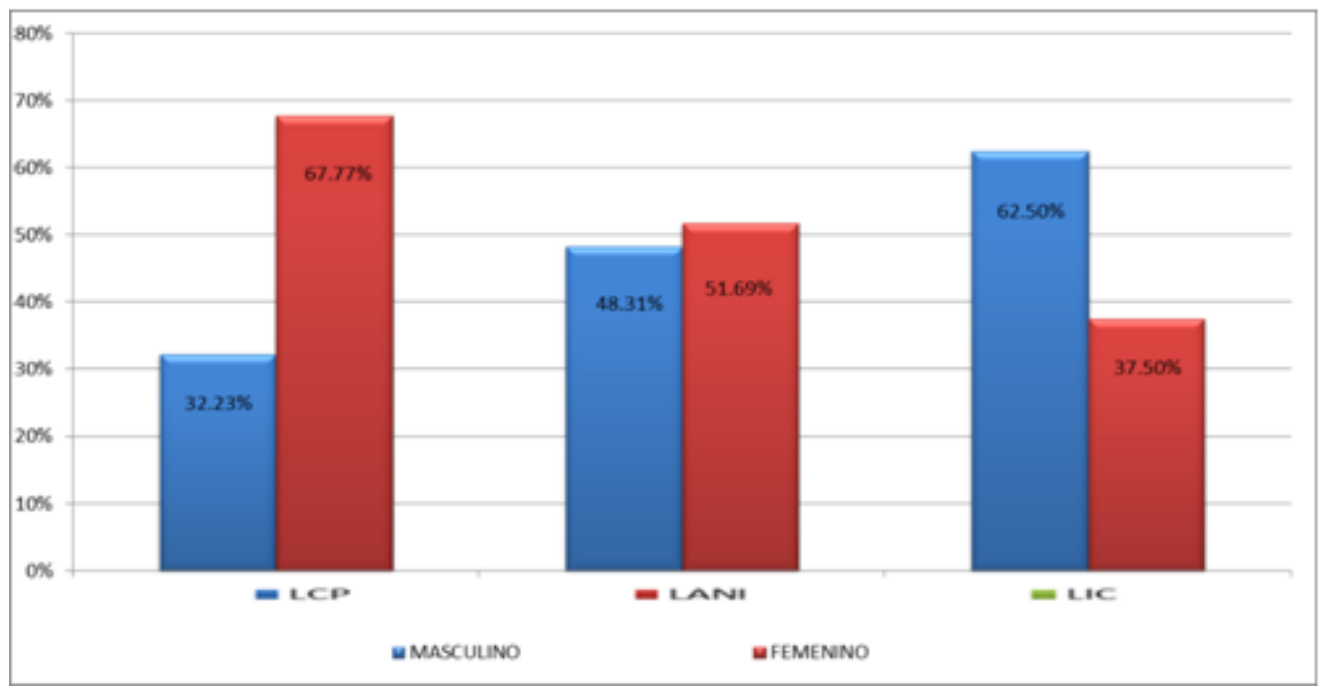

Gráfico 1. Frecuencia y género por especialidad

El mayor número de estudiantes es de Contabilidad 121 (48\%), seguido por 118 (46\%) de Administración y un número más pequeño de Comercial solo 16 (6\%). El género femenino es predominante en la muestra para un 58,43\% y el masculino $41,57 \%$.

Tabla 2. Distribución por edades y especialidad

\begin{tabular}{|c|c|c|c|c|c|c|c|c|}
\hline \multirow[t]{2}{*}{ Edades } & \multicolumn{2}{|c|}{ LCP } & \multicolumn{2}{|c|}{ LANI } & \multicolumn{2}{|c|}{ LIC } & \multirow[t]{2}{*}{ Total } & \multirow[b]{2}{*}{$\%$} \\
\hline & $f$ & $\%$ & & $\%$ & & $\%$ & & \\
\hline $16-20$ & 3 & $2,48 \%$ & 2 & $1,69 \%$ & 0 & $0,00 \%$ & 5 & $1,96 \%$ \\
\hline $21-25$ & 44 & $36,36 \%$ & 46 & $38,98 \%$ & 7 & $43,75 \%$ & 97 & $38,04 \%$ \\
\hline $26-30$ & 36 & $29,75 \%$ & 29 & $24,58 \%$ & 6 & $37,50 \%$ & 71 & $27,84 \%$ \\
\hline $31-35$ & 23 & $19,01 \%$ & 24 & $20,34 \%$ & 2 & $12,50 \%$ & 49 & $19,22 \%$ \\
\hline $35-40$ & 9 & $7,44 \%$ & 12 & $10,17 \%$ & 1 & $6,25 \%$ & 22 & $8,63 \%$ \\
\hline $41-45$ & 5 & $4,13 \%$ & 3 & $2,54 \%$ & 0 & $0,00 \%$ & 8 & $3,14 \%$ \\
\hline $46-50$ & 1 & $0,83 \%$ & 2 & $1,69 \%$ & 0 & $0,00 \%$ & 3 & $1,18 \%$ \\
\hline Total & 121 & $100,00 \%$ & 118 & $100,00 \%$ & 16 & $100,00 \%$ & 255 & $100,00 \%$ \\
\hline
\end{tabular}




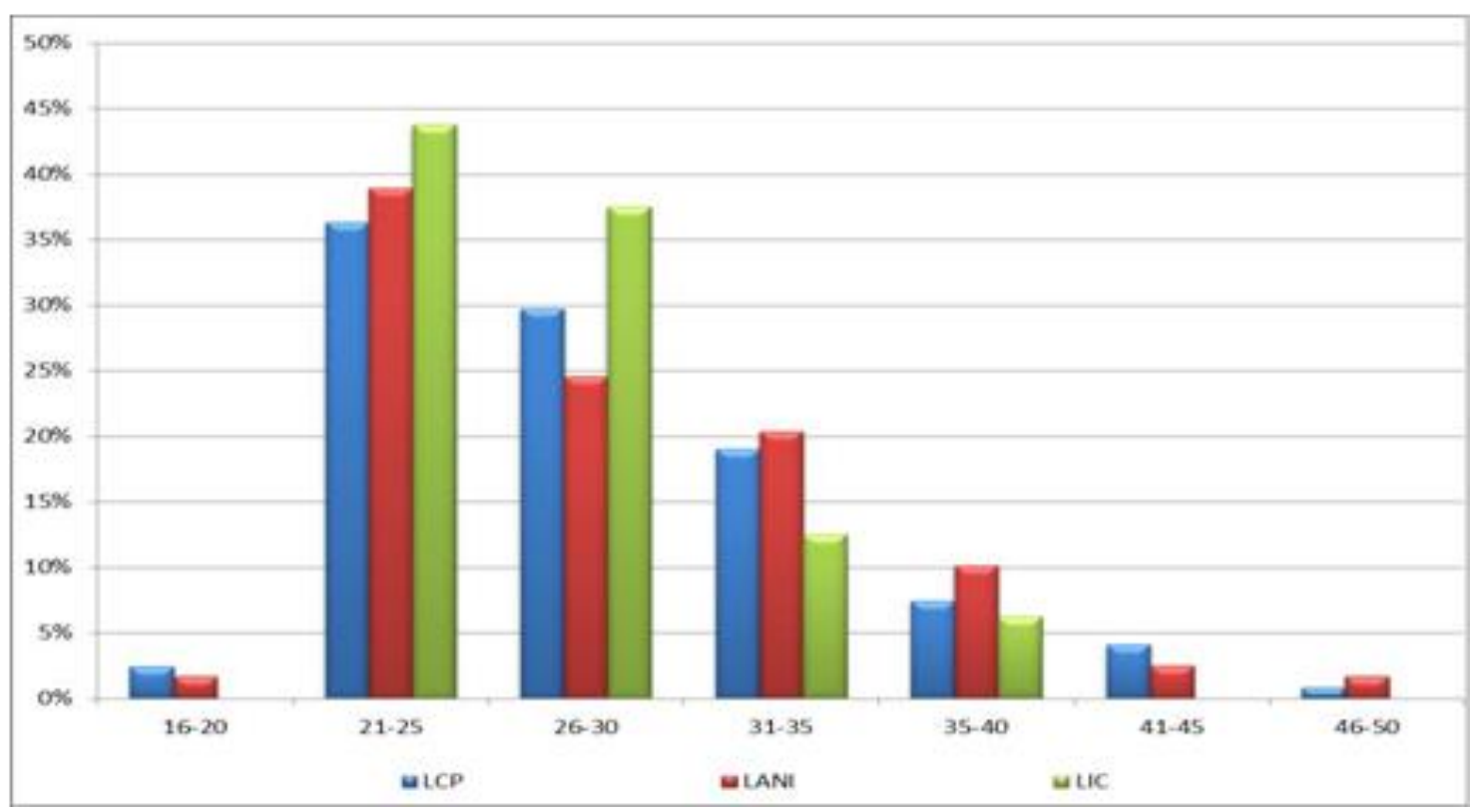

Gráfico 2. Distribución por edades y especialidad

El $65,88 \%$ de la muestra se encuentra distribuida entre los 21 y 30 años.

La distribución de acuerdo a la religión que profesan los estudiantes se presenta en la Tabla 3 y el Gráfico respectivo

Tabla 3. Distribución por religión

\begin{tabular}{lcccccccc}
\hline \multirow{2}{*}{ Religión } & \multicolumn{1}{c}{ LCP } & \multicolumn{2}{c}{ LANI } & \multicolumn{2}{c}{ LIC } & & Total \\
& $\mathbf{f}$ & $\mathbf{\%}$ & $\mathbf{f}$ & $\mathbf{\%}$ & $\mathbf{f}$ & $\mathbf{\%}$ & $\mathbf{f}$ & $\%$ \\
\hline Católico & $\mathbf{1 2}$ & $9,92 \%$ & $\mathbf{1 5}$ & $12,71 \%$ & $\mathbf{3}$ & $18,75 \%$ & 30 & $11,76 \%$ \\
Adventista & 52 & $42,98 \%$ & 48 & $40,68 \%$ & 7 & $43,75 \%$ & 107 & $41,96 \%$ \\
Evangélico & 26 & $21,49 \%$ & 28 & $23,73 \%$ & 3 & $18,75 \%$ & 57 & $22,35 \%$ \\
Mormón & 2 & $1,65 \%$ & 4 & $3,39 \%$ & 0 & $0,00 \%$ & 6 & $2,35 \%$ \\
Testigo de Jehová & 7 & $5,79 \%$ & 5 & $4,24 \%$ & 0 & $0,00 \%$ & 12 & $4,71 \%$ \\
Otra Cristiana & 18 & $14,88 \%$ & 15 & $12,71 \%$ & 2 & $12,50 \%$ & 35 & $13,73 \%$ \\
Otra No Cristiana & 4 & $3,31 \%$ & 3 & $2,54 \%$ & 1 & $6,25 \%$ & 8 & $3,14 \%$ \\
Total & $\mathbf{1 2 1}$ & $\mathbf{1 0 0 , 0 0 \%}$ & $\mathbf{1 1 8}$ & $\mathbf{1 0 0 , 0 0 \%}$ & $\mathbf{1 6}$ & $\mathbf{1 0 0 , 0 0 \%}$ & $\mathbf{2 5 5}$ & $\mathbf{1 0 0 , 0 0 \%}$ \\
\hline
\end{tabular}




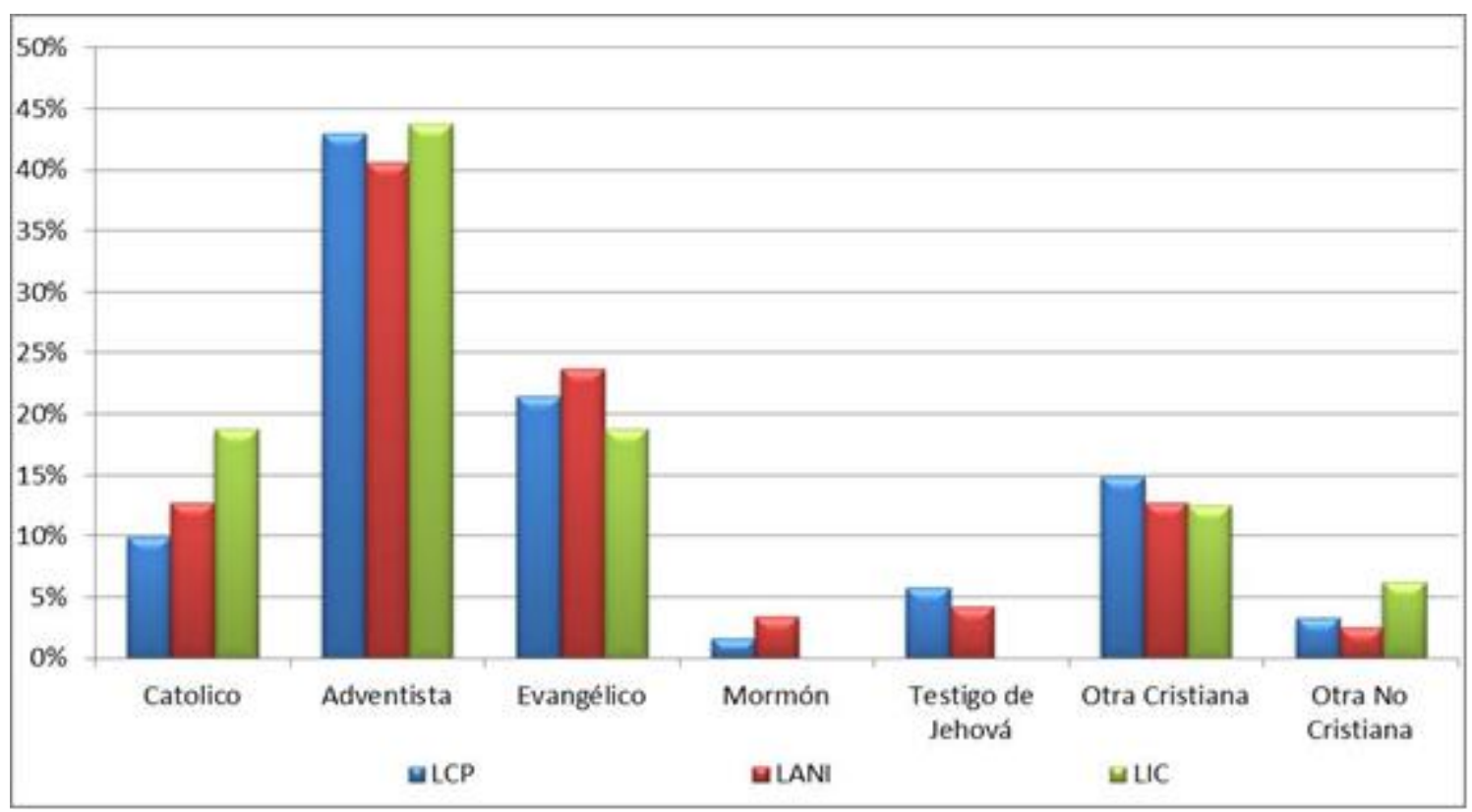

Gráfico 3. Distribución por religión

Los estudiantes de la muestra profesan la religión Adventista son 107 equivalen a 41,96\%; Evangélicos son el 22,35\% con 57 estudiantes, los que profesan otra religión Cristiana son el $13,73 \%$ con 35 , seguido por los Católicos con $11,76 \%$ con 30 estudiantes.

A continuación se presentan los resultados de los Factores y las Características en la Tabla 4 y Gráfico respectivo.

Tabla 4. Resumen General de los Factores y Características

\begin{tabular}{|c|c|c|c|c|c|c|c|c|}
\hline \multirow{2}{*}{$\begin{array}{l}\text { Factores y } \\
\text { aracterísticas }\end{array}$} & \multirow{2}{*}{$\begin{array}{l}\text { LCP } \\
\text { Nivel }\end{array}$} & \multirow[b]{2}{*}{$\%$} & \multirow{2}{*}{$\begin{array}{l}\text { LANI } \\
\text { Nivel }\end{array}$} & \multirow[b]{2}{*}{$\%$} & \multirow{2}{*}{$\begin{array}{l}\text { LIC } \\
\text { Nivel }\end{array}$} & \multirow[b]{2}{*}{$\%$} & \multicolumn{2}{|c|}{$\begin{array}{l}\text { Promedio } \\
\text { FCEA }\end{array}$} \\
\hline & & & & & & & Nivel & $\%$ \\
\hline \multicolumn{9}{|l|}{ F1: Características asociadas a la } \\
\hline Misión y al Proyecto Institucional & 3,54 & $70,70 \%$ & 3,54 & $70,72 \%$ & 3,72 & $74,38 \%$ & 3,60 & $71,93 \%$ \\
\hline \multicolumn{9}{|l|}{ F2: Características Asociadas a los } \\
\hline Estudiantes & 3,58 & $71,55 \%$ & 3,54 & $70,90 \%$ & 3,62 & $72,32 \%$ & 3,58 & $71,59 \%$ \\
\hline \multicolumn{9}{|l|}{ F3: Características Asociadas a los } \\
\hline $\begin{array}{l}\text { Profesores } \\
\text { F4: Características Asociadas a los }\end{array}$ & 3,16 & $63,14 \%$ & 3,14 & $62,87 \%$ & 3,06 & $61,25 \%$ & 3,12 & $62,42 \%$ \\
\hline Procesos Académicos & 2,99 & $59,88 \%$ & 3,09 & $61,74 \%$ & 3,29 & $65,77 \%$ & 3,12 & $62,46 \%$ \\
\hline $\begin{array}{l}\text { F5: Características Asociadas al } \\
\text { Bienestar Institucional }\end{array}$ & 3,98 & $79,66 \%$ & 3,94 & $78,84 \%$ & 4,13 & $82,50 \%$ & 4,02 & $80,33 \%$ \\
\hline
\end{tabular}




\begin{tabular}{ccccccccc}
\hline Factores y & LCP & & LANI & & LIC & & Promedio \\
Características & & & & & & & & \\
& & & & & & & & \\
& Nivel & $\%$ & Nivel & $\%$ & Nivel & $\%$ & Nivel & $\%$ \\
\hline
\end{tabular}

F6: Características Asociadas a la Organización, Administración y
Gestión
$3,19 \quad 63,81 \%$
3,12
$62,48 \%$
$3,38 \quad 67,50 \%$
$3,23 \quad 64,60 \%$

F7: Características Asociadas a los

Egresados e Impacto sobre el medio

$2,87 \quad 57,46 \% \quad 2,90 \quad 57,96 \% \quad 2,69 \quad 53,75 \% \quad 2,82 \quad 56,39 \%$

F8: Características Asociadas a los

Recursos Físicos y financieros

$3,22 \quad 64,35 \% \quad 3,39 \quad 67,71 \% \quad 3,23 \quad 64,58 \% \quad 3,28 \quad 65,55 \%$

Promedios Totales

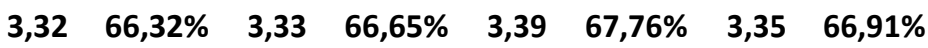

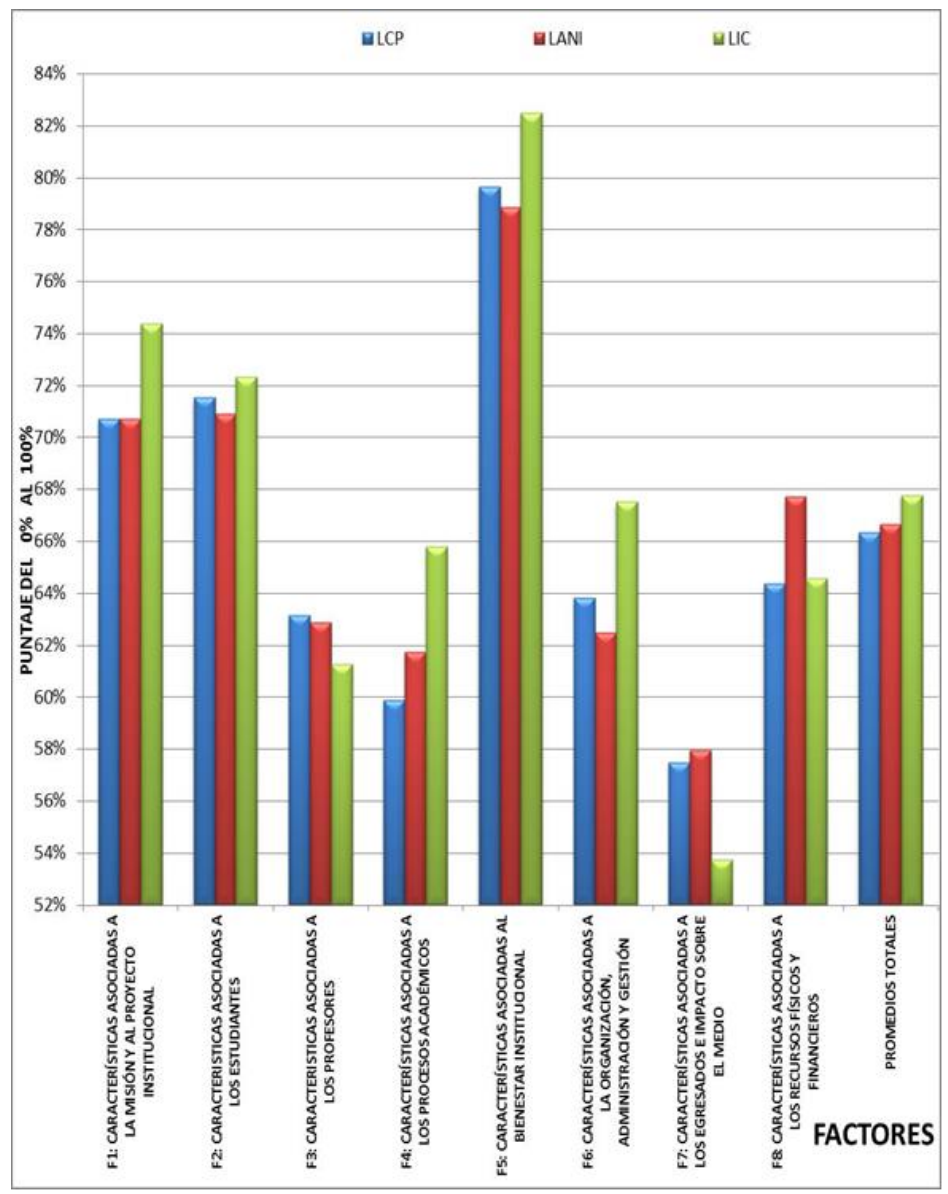

Gráfico 4. Resumen General de los Factores y Características 
La Tabla y el gráfico № 4, muestran los hallazgos de los Factores y sus puntajes acumulados en cada una de las características. En una escala del 1 al 5 la calificación que tuvo cada uno de sus componentes muestra la siguiente situación:

El factor 1: Características asociadas a la misión y al proyecto institucional. Obtuvo un promedio en su calificación del 3.60 y en la escala de porcentaje Comercial obtiene 74.38\%, Contabilidad 70.70\%, y Administración 70.72\%. El promedio general es $71.93 \%$, el factor 1 se califica en el Nivel 4 como: "Calidad con menor distancia con respecto al modelo considerado deseable".

El factor 2: Características asociadas a los estudiantes. Obtuvo un promedio en su calificación de 3.58 y en la escala de porcentaje Comercial obtuvo $72.32 \%$, Contabilidad $71.55 \%$ y Administración c70.90\%. El promedio general es $71.59 \%$, el factor 2 se ubica en el Nivel 4 como: "Calidad con menor distancia con respecto al modelo considerado deseable".

El factor 3: Características asociadas a los profesores. Obtuvo un promedio en su calificación de 3.12 y en la escala de porcentaje Comercial obtuvo $61.25 \%$, Contabilidad $63.14 \%$ y Administración $62.87 \%$. El promedio general es $62.42 \%$. El factor 3 se ubica en el Nivel 3 como: "Calidad como razonable con respecto al modelo considerado deseable".

El factor 4: Características asociadas a los procesos académicos. Obtuvo un promedio en su calificación de 3.12 y en la escala de porcentaje Comercial obtuvo 65.77\%, Contabilidad 59.88\% y Administración $61.74 \%$. El promedio general es el $62.46 \%$, el factor 4 se ubica en el Nivel 3 como: "Calidad como razonable con respecto al modelo considerado deseable".
El factor 5: Características asociadas al bienestar institucional. Obtuvo un promedio en su calificación del 4.02 y en la escala de porcentaje Comercial obtuvo 82.50\%, Contabilidad 79.66\%, y Administración 78.84\%. El promedio general es el $80.33 \%$, el factor 5 se ubica en el Nivel 4 como: "Calidad con menor distancia con respecto al modelo considerado deseable".

El factor 6: Características asociadas a la organización, administración y gestión. Obtuvo un promedio en su calificación de 3.23 y en la escala de porcentaje Comercial obtuvo 67.50\%, Contabilidad $63.81 \% \mathrm{y}$ Administración 62.48\%. El promedio general es el $64.60 \%$, el factor 6 se ubica en el Nivel 3 como: "Calidad como razonable con respecto al modelo considerado deseable".

El factor 7: Características asociadas a los egresados e impacto sobre el medio. Obtuvo un promedio en su calificación de 2.82 y en la escala de porcentaje Comercial obtuvo 53.75\%, Contabilidad 57.46\% y Administración 57.96\%. El promedio general es el $56.39 \%$, el factor 7 se ubica en el Nivel 3 como: "Calidad como razonable con respecto al modelo considerado deseable".

El factor 8: Características asociadas a los recursos físicos y financieros. Obtuvo un promedio en su calificación de 3.28 y en la escala de porcentaje Comercial obtuvo 64.58\%, Contabilidad $64.35 \% \quad \mathrm{y}$ Administración $67.71 \%$. El promedio general es el $65.55 \%$, el factor 8 se ubica en el Nivel 3 como: "Calidad como razonable con respecto al modelo considerado deseable".

El promedio general de la Facultad de Ciencias Económicas es de 3.35 y en la escala de porcentaje obtiene un promedio del 66.91\%. El promedio general de todos los factores en la FCEA se ubica en el Nivel 3 como: "Calidad como razonable con respecto al modelo considerado deseable". 


\section{CONCLUSIONES}

El factor 1: Características asociadas a la misión y al proyecto institucional, el factor 2 : Características asociadas a los estudiantes y el factor 5: Características asociadas al bienestar institucional; son los factores que obtuvieron mayor calificación se ubicaron en el Nivel 4 como: "Calidad con menor distancia con respecto al modelo considerado deseable". Los factores restantes se ubicaron todos en el Nivel 3, como: "Calidad como razonable con respecto al modelo considerado deseable".

Como fortalezas, en cuanto a la calidad educativa en la FCEA, se consideran en cada factor aquellas características que obtuvieron mayor calificación, así se tiene en el Factor 5: Características asociadas al bienestar institucional 80,33\%, que corresponde al nivel 4 , referente a las políticas, programas y servicios de bienestar universitario.

El Factor 1: Características asociadas a la misión y al proyecto institucional $71,93 \%$, que corresponde al nivel 4, referente a la: Misión Institucional, Proyecto Institucional, Proyecto Educativo del Programa y la Pertinencia del Programa.

Factor 2: Características asociadas a los estudiantes $71,59 \%$, correspondiente al nivel 4, referente a: Mecanismos de Ingreso, Numero de los estudiantes admitidos, Permanencia y deserción estudiantil, Participación en actividades de formación integral, Competencias, Reglamento estudiantil y Evaluación de estudiantes.

Factor 8: Características asociadas a los recursos físicos y financieros 65,55\%, nivel 3, correspondiente a: Recursos físico, Presupuesto del programa y Administración de recursos.

Los factores caracterizan y contribuyen significativamente con la calidad educativa de FCEA. Son los cuatro factores que se presentan en la anterior conclusión como fortalezas: Factor 5: Características asociadas al bienestar institucional (80,33\%, nivel 5); Factor 1: Características asociadas a la misión y al proyecto institucional (71,93\%, nivel 4); Factor 2: Características asociadas a los estudiantes (71,59\%, nivel 4); Factor 8: Características asociadas a los recursos físicos y financieros (65,55\%, nivel 3).

Entre las debilidades se tienen las menores puntuaciones en el factor 7 como las características asociadas a los egresados, el factor 3 relacionado con las características asociadas a los profesores y el factor 4 relacionado con las características de los proceso educativos. Cabe destacar que todos estos factores puntuaron para calificar en el Nivel 3, lo que indica que la calidad es aceptable: "Calidad como razonable con respecto al modelo considerado deseable".

Por último La hipótesis planteada en este estudio fue que el nivel promedio alcanzado en los factores y características que contribuyen con la calidad educativa de la FCEA de la UAB, desde la satisfacción de los estudiantes del plan de estudios 2016 - 2020, está en el Nivel 3 entre $56-70$, se cumple aceptablemente (Calidad como razonable con respecto al modelo considerado deseable).

La hipótesis es aceptada por que se encuentran todos los factores $\mathrm{y}$ características en la escala del Nivel 3, la calificación promedio general alcanzado por FCEA en esta evaluación alcanza al $66.91 \%$ que corresponde al nivel 3 (se cumple aceptablemente).

\section{REFERENCIAS}

Alarcón, J. (2008) Constructivismo y Calidad Educativa. UMBRAL, Revista de Educación, Cultura y Sociedad. 13(4) 
[Revista en línea]. Disponible: http://portal.fachse.edu.pe/sites/defa ult/files/U1314-a14.pdf

Bruner, J. (2008). El proceso de Bolonia en el horizonte latinoamericano: límites y posibilidades. Revista de Educación, número extraordinario, 119 - 145

Fernández, N. (2005). Los sistemas de evaluación y acreditación de la calidad y el desarrollo universitario: una visión latinoamericana comparada en los Procesos de Acreditación en el Desarrollo de las Universidades. CINDA, UNESCO-IESAL y Universidad de los Andes, Santiago de Chile

Lago de Vergara, D; Gamoba, A. y Montes, J. (2014) Calidad de la educación superior: un análisis de sus principales determinantes. SABER, CIENCIA $y$ Libertad. 8(2), 157 - 169

Lago, D., López, E., Municio, P., Ospina, R., y Vergara, G. (2013). La Calidad de la Educación Superior. ¿Un reto o una utopía? Bogotá: Ecoe

Luna, M. (1997). Introducción a los fundamentos teológicos y filosóficos de la educación religiosa. Entre Ríos, Argentina: Universidad Adventista del Plata
MERCOSUR. (2008) /CMC/DEC N $\mathrm{N}^{\circ}$ 17/08 Acuerdo sobre la creación e implementación de un Sistema de Acreditación de Carreras Universitarias para el Reconocimiento regional de la Calidad Académica de las respectivas titulaciones en el MERCOSUR y estados asociados [Documento en línea] San Miguel de Tucumán Recuperado de http://www.cartillaciudadania.mercos ur.int/oldAssets/uploads/DEC_0172008_ES_Acdo\%20Acreditacion\%20Carr eras\%20Universitarias.pdf

Unesco. (2009) Conferencia mundial sobre educación superior - 2009: La nueva dinámica de la educación superior y la investigación para el cambio social y el desarrollo. (Sede de la UNESCO, París, 5-8 de julio de 2009): Recuperado de http://www.unesco.org/education/WC HE2009/comunicado_es.pdfBanco Mundial, Tema educación: http://www.bancomundial.org/es/topi c/education/overviewOMC. Acuerdo General de Comercio de Servicios 